\title{
X-Ray Studies of the Pulsar Wind Nebula Around PSR B0540-69
}

\author{
R. Petre, U. Hwang \\ Laboratory for High Energy Astrophysics, NASA / GSFC, Greenbelt, \\ MD 20771, USA
}

S. S. Holt

F.W. Olin College of Engineering, Needham, MA 02492, USA

R. M. Williams

Department of Astronomy, University of Ilinois, Urbana, IL 61801, USA

\begin{abstract}
The pulsar wind nebula (PWN) surrounding the 50-ms pulsar B0540-69 in the Large Magellanic Cloud shares many properties with the Crab Nebula, the canonical Galactic PWN. We have used the ACIS instrument on the Chandra X-ray Observatory to perform spatially resolved spectroscopy of the PWN. The spectrum of the inner $10^{\prime \prime}$ is characterized by a simple power law, with a spectral index that steepens strongly with radius. Outside the central $10^{\prime \prime}$ the spectra also require a thermal component. This diameter corresponds to a shell detected in [O III], suggesting that this shell represents a skin around the PWN, in analogy to the Crab. We infer the value of several key PWN parameters, including $\sigma$, the average ratio of electromagnetic to particle flux.
\end{abstract}

\section{Introduction}

The supernova remnant (SNR) 0540-69.3 is the second youngest SNR in the Large Magellanic Cloud (LMC) after SN 1987A, and contains the LMC's most luminous pulsar wind nebula (PWN). This PWN, containing a 50-ms pulsar, has been called the "twin" of the Crab: it has similar age, overall morphology, ratio of X-ray luminosity to spin down energy, age, and optical properties. It has some notable differences as well, including smaller spin-down luminosity and braking index, and most significantly the presence of a shell of shock-heated gas surrounding the PWN. Thus SNR 0540-69.3 is at best a fraternal twin of the Crab. To investigate the thermal X-ray emission from the shell and to perform spatially resolved spectroscopy of the PWN, we carried out a 27-ks ACIS observation. The analysis of the shell has been reported by Hwang et al. (2001); here we summarize our results on the PWN. 


\section{Results}

Figure 1 shows the ACIS image of SNR 0540-69.3. The PWN has a substantially higher surface brightness than the thermal shell. In Figure 2 we show the HRC and ACIS images of the PWN. A comparison between the two upper figures reveals the depressed surface brightness at the center of the PWN in the ACIS image caused by pulse pile-up. In the lower twp panels we overlay the HRC (left) and ACIS (right) contours on an HST [O III] image. The [O III] shell has comparable dimension and shape to that of the Chandra images.

We extracted spectra from eight elliptical regions centered on the pulsar. The bin width for the seven inner regions is 0.5 , the resolution of ACIS. The seventh and eighth radial bins have widths of $1{ }^{\prime \prime} 0$ and $1 . \prime 5$. The pulsar spectrum was extracted from the pulse pile-up trail. The spectra from the innermost seven regions (six ellipses plus the pular) were well fit with an absorbed power law $\left(\chi_{\nu}^{2} \sim 1\right)$. The photon index varies smoothly with radius from 1.3 to 2.4 ; the spectrum of the pulsar is considerably softer $(\Gamma \sim 1.95 \pm 0.15)$. For each region, $N_{H} \sim 5 \times 10^{21} \mathrm{~cm}^{-2}$. The two outer spectra require a thermal component in addition to a power law. As indicated in the left panel of Figure 3, this spectral character change occurs at the radius of the [O III] shell.

We determined the nebular size as a function of energy by creating six narrow band images over the 0.8 $10.0 \mathrm{keV}$ energy range and fitting each using a 2D gaussian. In the right panel of Figure 3, we plot the average radius as a function of energy. The data are fit by a power law of the form $R(E)=10 E^{(-0.18 \pm 0.02)}$ arcsec. The energy dependent nebular size is presumably due to the synchrotron "burn off" of high energy electrons.

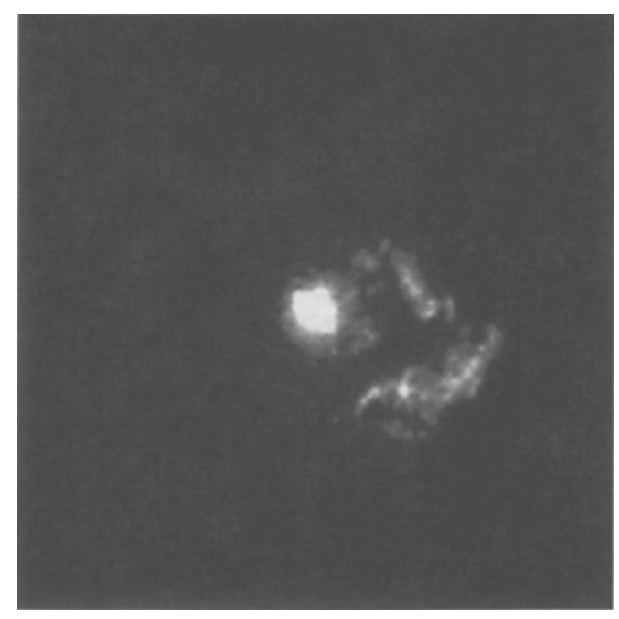

\section{Discussion}

We have used X-ray, optical and radio data to infer and compile parameters for the PWN. These are compared with those of the Crab in Table 1 . Some of the key results from our analysis are as follows. (i) The X-ray nebulosity is comparable in

Figure 1. ACIS image of SNR 054069.3. The pulsar wind nebula, overexposed in this rendition, is embedded in the center of a thermal shell. Faint streaks toward the northwest and southeast are artifacts of pulse pile-up from the pulsar. 


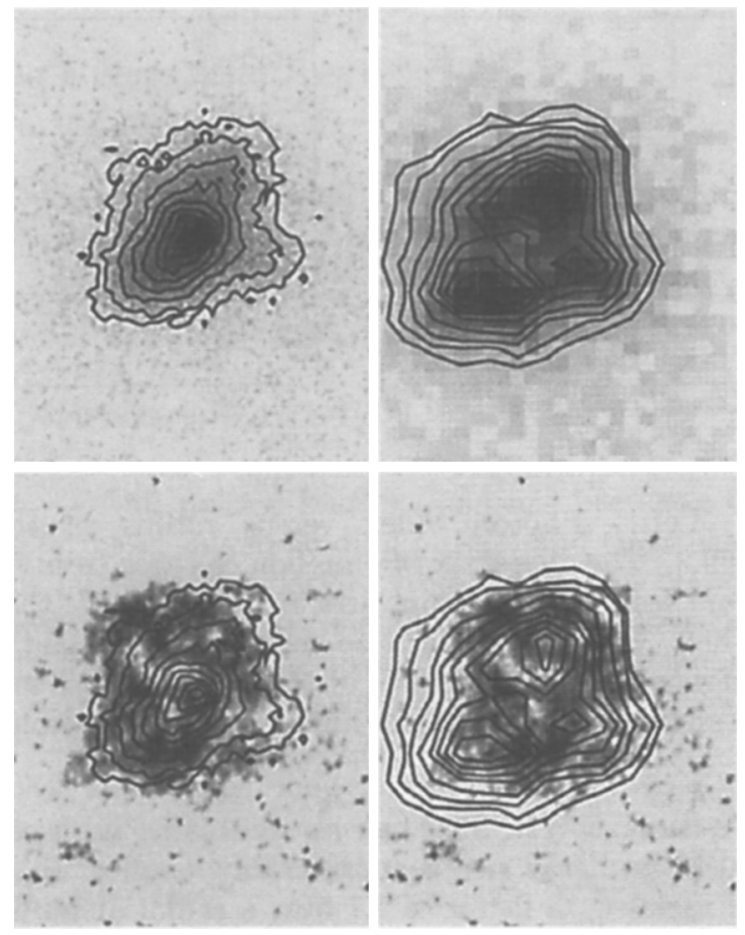

Figure 2. Images of the synchrotron nebula in SNR 0540-69.3. Top left: HRC image. Top right: ACIS image. Bottom left: HRC contours overlaid on HST [O III] image. Bottom right: ACIS contours overlaid on $H S T$ [O III] image. Comparison between the HRC and ACIS images indicates the influence of pulse pile-up on the center of the ACIS image.

Table 1. Comparison of properties of the PWN in SNR 0540-69.3 with those of the Crab Nebula.

\begin{tabular}{lcc}
\hline Parameter & $0540-69.3$ & Crab \\
\hline X-ray size (pc) & $1.2 \times 0.7$ & 1.2 \\
Radio size (pc) & $1.4 \times 1.3$ & $3.5 \times 2.3$ \\
Distance (kpc) & 50 & 2 \\
Age $(\mathrm{yr})$ & $800-1000$ & 950 \\
$L_{x}\left(\mathrm{ergs} \mathrm{s}^{-1}\right)$ & $8 \times 10^{36}$ & $2.1 \times 10^{37}$ \\
$\dot{E}\left(\mathrm{ergs} \mathrm{s}^{-1}\right)$ & $1.5 \times 10^{38}$ & $4.7 \times 10^{38}$ \\
$L_{x} / \dot{E}$ & 0.05 & 0.05 \\
$\Gamma$ range & $1.3-2.4$ & $1.6-2.3$ \\
$\sigma$ & $(2.5-9) \times 10^{-3}$ & $3 \times 10^{-3}$ \\
\hline
\end{tabular}



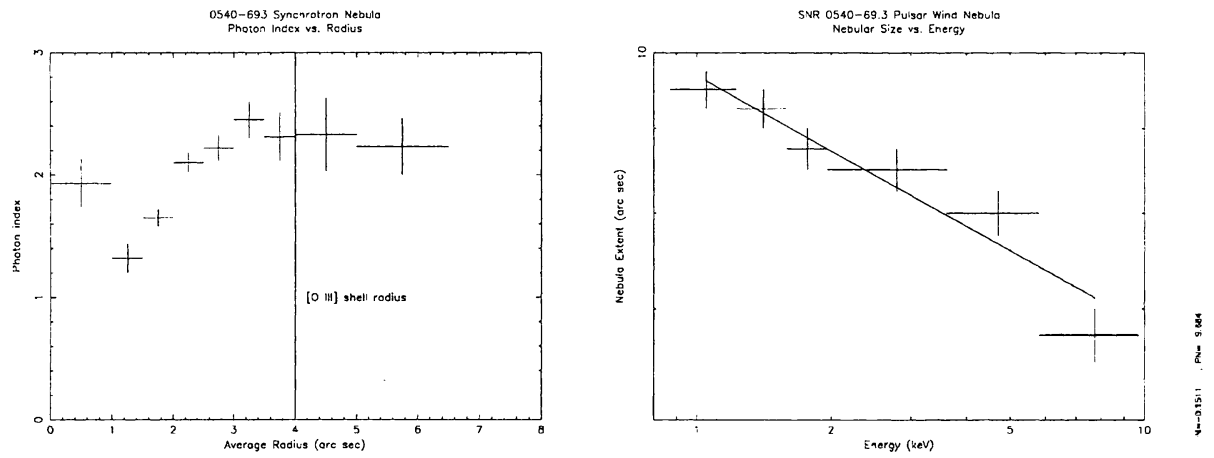

Figure 3. Left: Photon index versus radius in the PWN in SNR 0540-69.3. The character of emission changes from a pure power law to a more complex spectrum at a radius of $\sim 5^{\prime \prime}$, the location of the [O III] shell. Right: Nebular size versus energy. The reduced size at higher energies is a consequence of the shorter synchrotron lifetime of the electrons responsible for the higher energy emission.

size to that of the radio PWN. This can be contrasted with other PWNe (e.g., the $\mathrm{Crab}$ ) in which the X-ray size is considerably smaller. (ii) The spectral index of the PWN varies by a factor of 1.1 over a radial distance of $1.2 \mathrm{pc}$. This implies more rapid evolution of the electron population than can be supported by a simple, one-zone magnetic field model. The magnetic field must decrease with radius. (iii) The character of the spectrum changes dramatically at a location corresponding to the [O III] shell. This suggests that the shell represents an outer "skin" as found in the Crab, where the PWN is pushing against the denser ejecta. (iv) Using a magnetic field strength of $8 \times 10^{-4} \mathrm{G}$ (Reynolds 1985), we estimate a synchrotron lifetime of $\sim 2 \mathrm{yr}$ for $0.5-\mathrm{keV} \mathrm{X}$-rays. Thus the electron streaming velocity is of order $0.5 c$. (v) The low value of $\sigma$, the ratio between the magnetic and particle flux, is comparable to that of the Crab, and shows that the nebula is particle dominated.

\section{References}

Hwang, U., Petre, R., Holt, S. S., \& Szymkowiak, A. E. 2001, ApJ, 560, 742 Reynolds, S. P. 1985, ApJ, 291, 152 